\title{
Localization of the large-angle foil-scattering beam loss caused by the multiturn charge-exchange injection
}

\author{
Shinichi Kato \\ Tohoku University, Sendai 980-8578, Japan
}

Kazami Yamamoto, Masahiro Yoshimoto, Hiroyuki Harada, and Michikazu Kinsho

Japan Atomic Energy Agency (JAEA), Tokai, Ibaraki, 319-1195, Japan

(Received 7 November 2012; published 31 July 2013)

\begin{abstract}
In the $3 \mathrm{GeV}$ rapid cycling synchrotron of the Japan Proton Accelerator Research Complex, significant losses were observed at the branching of the $\mathrm{H}^{0}$ dump line and the beam position monitor that was inserted downstream of the $\mathrm{H}^{0}$ dump branch duct. These losses were caused by the large-angle scattering of the injection and circulating beams at the charge-exchange foil. To realize high-power operation, these losses must be mitigated. Therefore, a new collimation system was developed and installed in October 2011. To efficiently optimize this system, the behavior of particles scattered by the foil and produced by the absorber were simulated, and the optimal position and angle of the absorber were investigated. During this process, an angle regulation method for the absorber was devised. An outline of this system, the angle regulation method for the absorber, and the performance of this new collimation system are described.
\end{abstract}

DOI: $10.1103 /$ PhysRevSTAB.16.071003

PACS numbers: 29.27.Ac

\section{INTRODUCTION}

For the proton synchrotron, the high beam intensity can be achieved by dividing the injection into a number of turns and adding the injection beam to the circulating beam. However, it is impossible to lead the injection beam to the same phase space which the circulating beam fills at the injection point if the injection beam and the circulating beam have the same charge. This principle is known as Liouville's theorem. To avoid this problem, $\mathrm{H}^{-}$, which has opposite charge to the circulating beam, is often used as the injection beam. In addition, the charge-exchange foil is placed at the injection point in order to convert $\mathrm{H}^{-}$ions to protons. This scheme is known as multiturn chargeexchange injection.

In this scheme, the circulating beam also collides with the foil. Although the foil is very thin and the energy of particles that collide with it is generally very high, it is expected that these particles are scattered and cause activation at the downstream area for high intensity accelerators.

The $3 \mathrm{GeV}$ rapid cycling synchrotron (RCS) of the Japan Proton Accelerator Research Complex (J-PARC) [1] serves as a proton beam source for the Materials \& Life Science Experimental Facility (MLF) and the $50 \mathrm{GeV}$ main ring (MR). The RCS has been providing a $3 \mathrm{GeV}$ proton beam to the MLF since 2008 and during this time the beam intensity was increased through a beam study. In February 2011 the RCS provided a $220 \mathrm{~kW}$ proton beam to

Published by the American Physical Society under the terms of the Creative Commons Attribution 3.0 License. Further distribution of this work must maintain attribution to the author(s) and the published article's title, journal citation, and DOI. the MLF; through further studies and upgrades we aim to achieve a $1 \mathrm{MW}$ beam power.

The RCS uses a multiturn charge-exchange injection scheme with $\mathrm{H}^{-}$beams [2]. The layout of the injection area is shown in Fig. 1. In the horizontal plane during the $500 \mu \mathrm{s}$ injection period, four shift-bump magnets (SB1-4) shape the fixed orbit bump and four paint-bump magnets (PB1-4) shape the time-decay orbit bump. $181 \mathrm{MeV} \mathrm{H}^{-}$ beams from the linac are transported to the injection point, stripped of electrons and converted to $\mathrm{H}^{+}$by the chargeexchange foil, and then injected into the ring, which is divided into 235 turns. The unstripped beams $\left(\mathrm{H}^{0}\right.$ and $\left.\mathrm{H}^{-}\right)$ are then stripped to $\mathrm{H}^{+}$by the 2nd and the 3rd foils downstream of the injection point and led to the $\mathrm{H}^{0}$ dump. During multiturn injection, the track of $\mathrm{H}^{-}$beams is fixed and the central orbit is shifted slightly from the charge-exchange foil by decreasing the time-decay orbit bump height. Therefore, the number of charge-exchange foil hits with circulating beams is decreased and the horizontal phase space of the ring is filled with the injection beams. It should be noted that the injection energy will be upgraded to $400 \mathrm{MeV}$ in the summer of 2013 to achieve higher intensity.

The charge-exchange foil is expected to heat up to over $2000 \mathrm{~K}$ during injection at $1 \mathrm{MW}$ operation. Therefore, development of the foil continues so that its lifetime under high temperatures can be improved. In the RCS, the hybrid boron mixed carbon foil that consists of $80 \%$ carbon and $20 \%$ boron is adopted [3].

The thickness of the foil was decided from the requirement of the charge-exchange efficiency. The injection beam becomes $\mathrm{H}^{0}$ which is an excited state when only one electron is stripped from $\mathrm{H}^{-}$. In the RCS, this $\mathrm{H}^{0}$ 


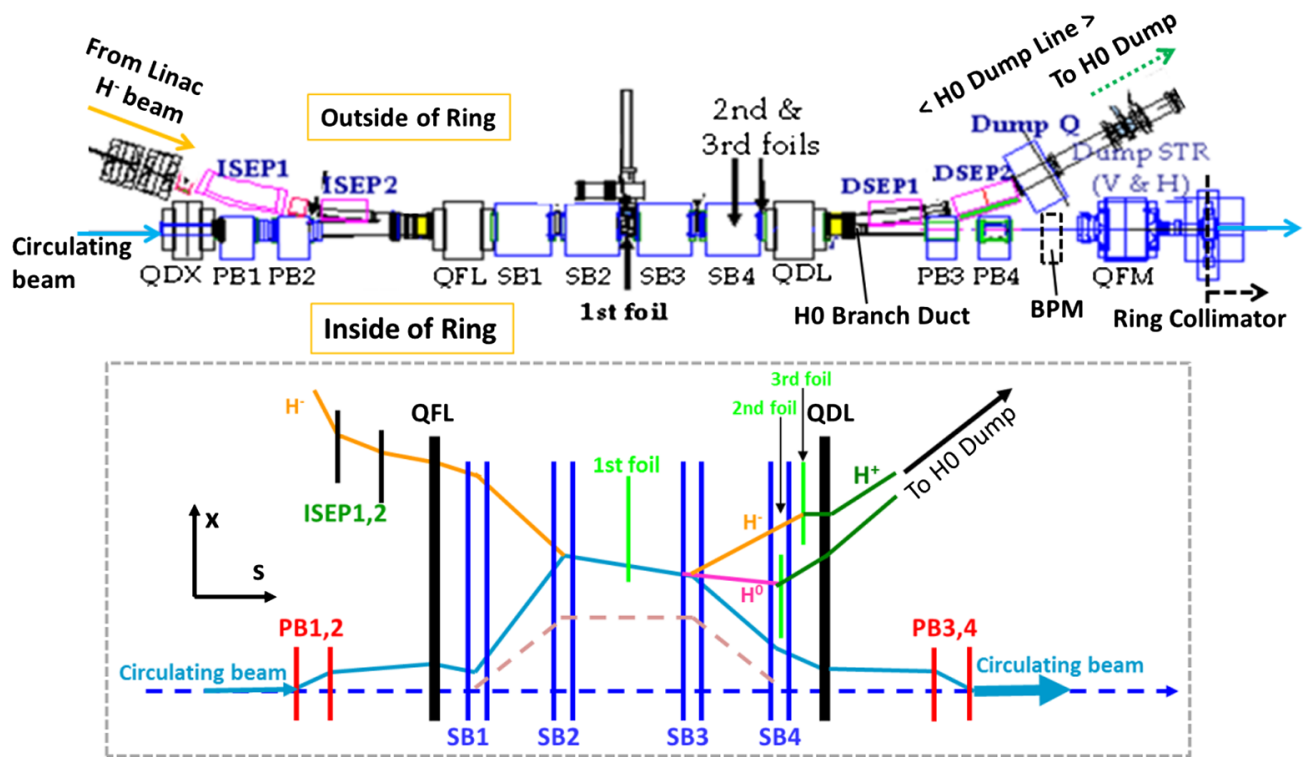

FIG. 1. Layout of the injection area and its schematic view.

causes uncontrollable beam loss when the $\mathrm{H}^{0}$ is broken down into $\mathrm{H}^{+}$by the peripheral magnetic field before reaching the 2 nd foil. Therefore, the charge-exchange efficiency was chosen so that the beam loss caused by $\mathrm{H}^{0}$ was $1 \mathrm{~W} / \mathrm{m}$ or less. In the RCS, the foil thickness is $200 \mu \mathrm{g} / \mathrm{cm}^{2}$ for the injection energy of $181 \mathrm{MeV}$ in order to achieve over $99.6 \%$ charge-exchange efficiency; a $300 \mu \mathrm{g} / \mathrm{cm}^{2}$ foil will be adopted for the planned injection energy of $400 \mathrm{MeV}$.

The RCS beam commissioning proceeded successfully [4]; however, after continuous user operation, unexpected losses were observed between the $\mathrm{H}^{0}$ branch duct and the horizontally focusing quadrupole magnet located downstream of the injection point (QFM). In particular, the residual doses were $4.5 \mathrm{mSv} / \mathrm{h}$ at the branching of the $\mathrm{H}^{0}$ dump line and $6.2 \mathrm{mSv} / \mathrm{h}$ at the inside of the beam position monitor (BPM). The BPM was inserted downstream of the $\mathrm{H}^{0}$ branch duct after a 4 hours cooling down period following one $220 \mathrm{~kW}$ cycle operation. These losses could not be ignored considering the exposure to radiation they caused during maintenance. From the results of some experiments and simulations, it became clear that these significant losses were caused by large-angle scattering of the injection and circulating beams at the charge-exchange foil during multiturn injection $[5,6]$.

Because the number of foil hits increases with increasing beam power, this problem must be solved to realize a much higher power operation. However, it is impossible, in principle, to completely prevent foil scattering. Therefore, an additional collimation system was developed to locally collect the large-angle-scattered particles and restrict the activated area [7]. First, based on simulation results, the area in which the particles were efficiently localized was estimated to be the area surrounding the $\mathrm{H}^{0}$ branch duct. A new $\mathrm{H}^{0}$ branch duct was therefore developed as the collimation system and integrated with two absorbers. Second, the $\mathrm{H}^{0}$ branch duct and absorbers were designed. Because the $\mathrm{H}^{0}$ branch duct requires magnetic shielding, this issue and the vacuum properties were also examined. Third, a simulation model was constructed to investigate the dependence of the localization efficiency on the absorber angle. Based on the results of this simulation, an angle regulation method for the absorbers was designed to maintain efficient localization. Finally, a beam test was performed, and the loss reduction was confirmed.

Herein, a reduction method for the loss caused by largeangle scattered particles using an additional collimation system is described, and the results are reported.

\section{H ${ }^{0}$ COLLIMATION SYSTEM}

\section{A. Structure of the $\mathbf{H}^{\mathbf{0}}$ collimation system}

A simulation of the paths of the scattered particles between the injection point and the ring collimator was performed to understand the behavior of the large-anglescattered particles and to estimate a suitable point for the localization of these particles. The results of this simulation are shown in Fig. 2.

In the injection area, the QDL, which is a horizontally defocusing quadrupole magnet, is installed upstream of the $\mathrm{H}^{0}$ branch duct. The scattered particles are subjected to the influence of the QDL, which increases the horizontal angle. Therefore, the source of these losses is the nearly scattered particles that have a large horizontal angle. The large-angle scattered particles are lost at the $\mathrm{H}^{0}$ branch and the BPM, which has a small aperture. These points agree with the high residual dose points found in practice. As a result of the tracking simulation, it became clear that the minimum horizontal scattering angle at which particles might be lost was approximately $\pm 3 \mathrm{mrad}$, while the 


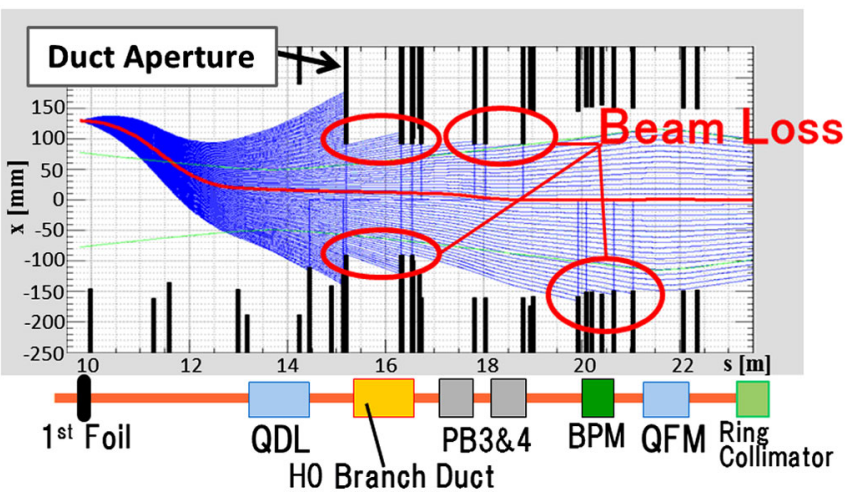

FIG. 2. Tracking simulation of the horizontally scattered beam. The blue lines are the tracks of particles that gave arbitrary angles at the injection point. The green lines indicate the $486 \pi \mathrm{mm}$ mrad beam envelope.

minimum vertical scattering angle at which particles might be lost was approximately $\pm 10 \mathrm{mrad}$.

The scattering simulation results of the GEANT4 [8] are shown in Fig. 3. About $10^{4}$ events that had a scattering angle exceeding $\pm 3 \mathrm{mrad}$ were obtained for the first time by increasing the simulation events up to $10^{9}$. These events were caused by the multiple Coulomb scattering and the energy loss hardly results. Although the probability of the occurrence of such large-angle scattering cases is very low, approximately $10^{-5}$, the total losses between the 1st foil and the ring collimator are estimated to be, at most, several $\mathrm{W}$ for a $200 \mathrm{~kW}$ operation due to repeated collisions of the circulating beam with the foil during the multiturn injection. In addition, when the injection energy is upgraded to $400 \mathrm{MeV}$, the number of events with a scattering angle of over $\pm 3 \mathrm{mrad}$ will decrease to only $80 \%$ of the $181 \mathrm{MeV}$ case. Therefore, the total losses will be expected to increase by a factor of 9 at $1 \mathrm{MW}$ operation due to the increase in kinetic energy and peak current compared to the current $200 \mathrm{~kW}$ operation.

With respect to the localization point, either more downstream or upstream of the loss points is a suitable

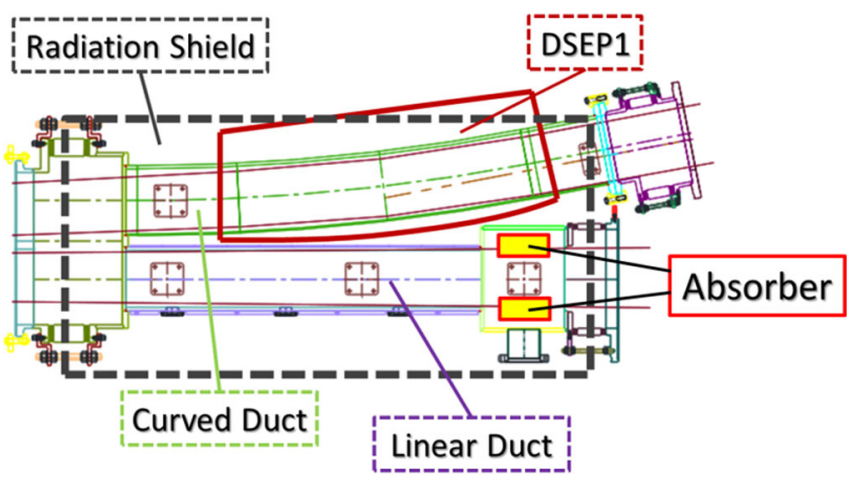

FIG. 4. Construction of the $\mathrm{H}^{0}$ collimation system.

localization point, because the particles that have large horizontal angles are more separated from the core beam downstream. However, the loss at the $\mathrm{H}^{0}$ branch cannot be localized upstream because the upstream magnet arrangement is too tight. Therefore, a new $\mathrm{H}^{0}$ branch duct was developed as the new collimation system.

This system consists of three elements: the $\mathrm{H}^{0}$ branch duct, two absorbers, and a radiation shield. The construction of this system is shown in Fig. 4. The $\mathrm{H}^{0}$ branch consists of a curved duct connected to the $\mathrm{H}^{0}$ dump line and a linear duct connected to the ring. Two absorbers were added at the downstream end of the linear duct to remove the particles that would otherwise have been lost between the absorbers and ring collimator. The radiation shield covers the $\mathrm{H}^{0}$ branch duct and the dump septum magnet (DSEP1), enabling maintenance in this area. The shield width is $450 \mathrm{~mm}$ and was selected on the basis of simulation results using the MARS [9] particle reaction simulation code. The simulation indicated that the residual dose on the shield surface would be less than $1 \mathrm{mSv} / \mathrm{h}$ after one operation cycle if the amount of beam loss at the absorber was $100 \mathrm{~W}$ [7]. The entire system, including the DSEP1, is installed on a linear motion guide and can thus be readily removed if it needs to be replaced.
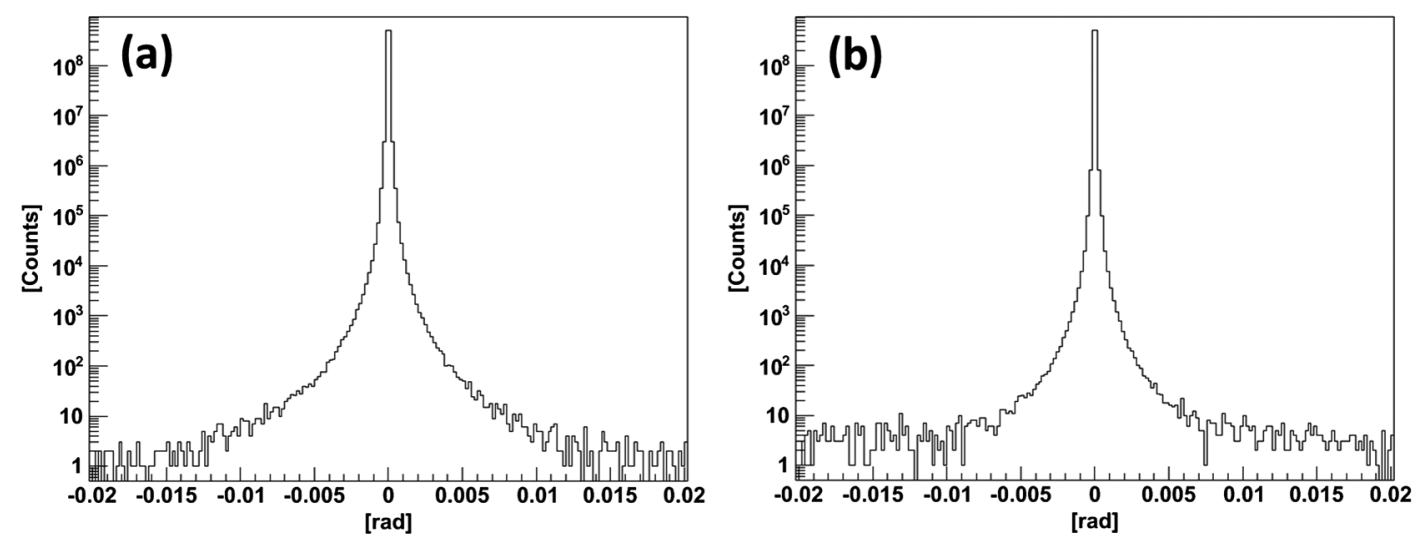

FIG. 3. The proton scattering distribution by the charge-exchange foil at (a) $181 \mathrm{MeV}$ with $200 \mu \mathrm{g} / \mathrm{cm}^{2}$ foil thickness, and (b) $400 \mathrm{MeV}$ with $300 \mu \mathrm{g} / \mathrm{cm}^{2}$ foil thickness. 


\section{B. Design of the $\mathbf{H}^{0}$ branch duct}

To suppress the perturbation of the circulation beams, the $\mathrm{H}^{0}$ branch duct needs to reduce the magnetic field due to the DSEP1 in the linear duct. However, there is not enough space to insert a shield that could screen the fringe field of the DSEP1. Therefore, it was decided that a linear duct was developed using magnetic stainless steel. However, such stainless steel is typically expensive especially as it is usually an article that is made to order. In particular, as the $\mathrm{H}^{0}$ collimation system is ultimately disposable, it was required that the material was inexpensive and readily available. Therefore, SUS430 was adopted as it is not only inexpensive and readily available, but its processing method is so well known that it is common to find it used as a kitchen table. SUS 430 consists of $0.60 \%$ or less of nickel compared to $10 \%$ found in common stainless steel which has no magnetic properties.

To realize a high-intensity-beam power, this duct must not only have a magnetic shielding effect but also low outgassing properties. However, data for the outgassing rate of SUS430 was scarce, because it has rarely been used for vacuum chambers. Therefore, the magnetic shielding effect and outgassing rate of a SUS430 linear duct were examined [10,11].

\section{Examination of the shielding effect}

A test duct was prepared from SUS430, and the magnetic shielding ability was examined by measuring the magnetic field inside the duct after the application of an external magnetic field. The test duct was a 500 -mm-long straight duct with a $60 \mathrm{~mm} \times 60 \mathrm{~mm}$ rectangular cross section and a $10 \mathrm{~mm}$ thickness. It was constructed to approximately $1 / 3$ scale of the linear section of the $\mathrm{H}^{0}$ branch duct, but with the same thickness. Because it had been reported that magnetic annealing could recover the magnetic permeability of SUS430 which was decreased by processing [10], two test ducts were prepared and compared by changing the magnetic annealing conditions as follows: heating for 4 hours at $850^{\circ} \mathrm{C}$ and no heating.

Based on the measurement results taken in 2006 when the DSEP1 was installed in the RCS [12], it was recognized that the leakage field caused by the DSEP1 was approximately several $\mathrm{mT}$. Therefore, Helmholtz coils that can generate a maximum field of approximately $8.5 \mathrm{mT}$ were prepared as the external magnetic source. The test duct was installed between the coils, and the magnetic field inside the test duct was measured with a one-dimensional Hall probe while changing the external magnetic field. The results are shown in Fig. 5.

As can be seen in the figure, the shielding efficiency of SUS430 without annealing was approximately 7\%, while after annealing it was approximately $2 \%$, and the magnetic field inside the duct was at most approximately $0.1 \mathrm{mT}$. Assuming that the magnetic field was distributed over the linear section of the $\mathrm{H}^{0}$ branch duct, the closed orbit

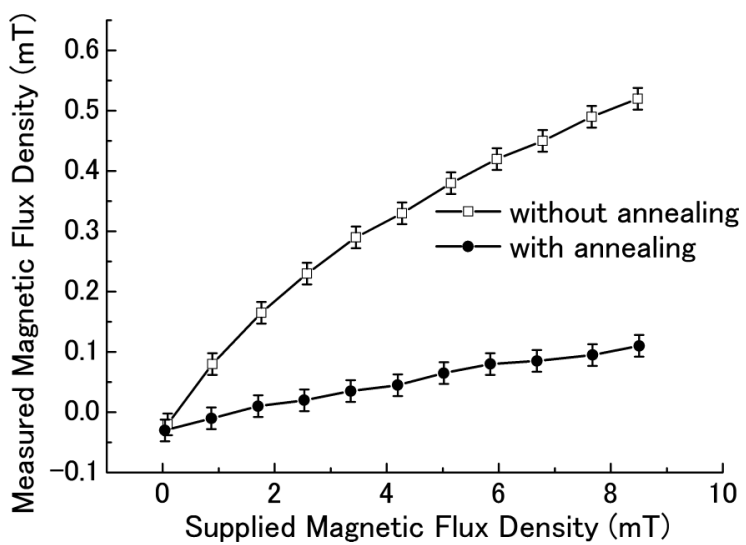

FIG. 5. Annealing effect of the SUS430. The horizontal axis represents the supplied magnetic flux density from Helmholtz coils, and the vertical axis represents the magnetic flux density measured with a Hall device probe.

distribution (COD) was calculated to be at most approximately $0.1 \mathrm{~mm}$, which can be corrected by the steering magnet. Thus, it was confirmed that an SUS430 duct has sufficient magnetic shielding ability for the linear section of the $\mathrm{H}^{0}$ branch duct.

\section{Examination of the vacuum properties}

In the RCS, the vacuum chambers have been designed such that their outgassing rates are sufficiently low to enable ultrahigh vacuum [13]. For example, the outgassing rate of the $\mathrm{Ti}$ bellows and ducts is approximately $10^{-9} \mathrm{~Pa} \mathrm{~m} / \mathrm{s}$, while that of the ceramic chamber is approximately $10^{-8} \mathrm{~Pa} \mathrm{~m} / \mathrm{s}$. For this reason, the outgassing rate of the new $\mathrm{H}^{0}$ branch duct must have a similar value. Therefore, the outgassing rate of SUS430 was determined by applying the differential pressure flow method to small sample plates treated in the same manner as the linear section of the new duct.

The outgassing rate of SUS430 was measured by comparing that of SUS304, which is a commonly used material for vacuum chambers, rather than to measure the absolute value. The outgassing rate of SUS304 has been reported to

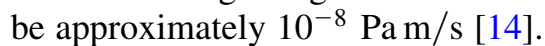

As a result of the measurement, it was determined that the outgassing rate of SUS430 was approximately 2 times that of SUS304 [11]. Therefore, it was concluded that SUS430 can be used as a material for vacuum chambers and the linear section of the new branch duct for installation in the RCS.

In addition, the outgassing rate of the constructed $\mathrm{H}^{0}$ duct was also measured and found to be $5.80 \times$ $10^{-9} \mathrm{~Pa} \mathrm{~m} / \mathrm{s}$. Therefore, it was confirmed that the outgassing rate of the new $\mathrm{H}^{0}$ branch duct is sufficiently low to be installed in the RCS.

\section{Design of the absorbers}

The absorbers must also meet certain requirements. First, they must have a low outgassing rate and enhanced 


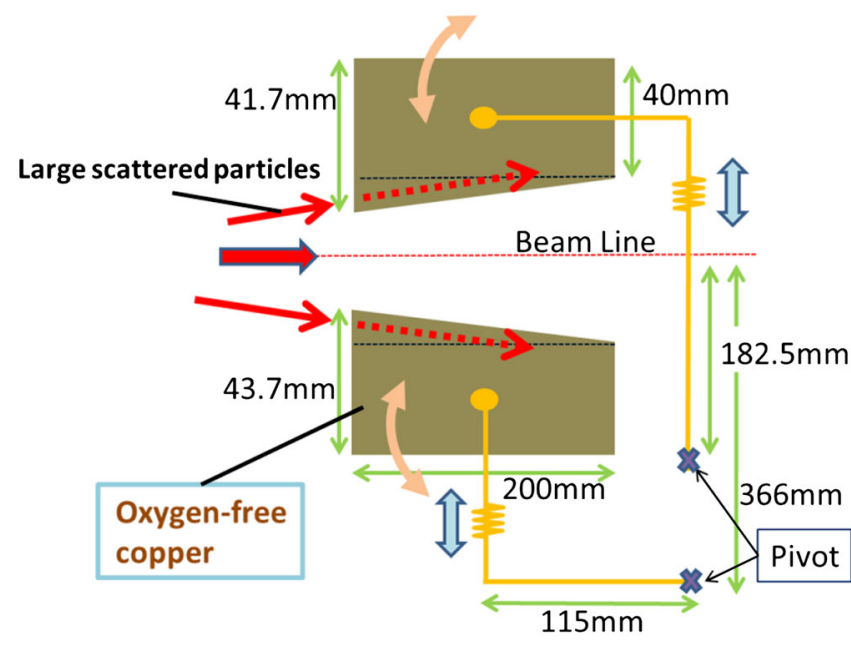

FIG. 6. Schematic drawing of the absorber.

thermal conduction for cooling. Therefore, oxygen-free copper, which was used as the ring collimator in the RCS [15], was selected. In addition, it was cooled naturally in air, because it was confirmed by finite element method calculations that the increase in the absorber temperature would be 80 degrees at most if the amount of the lost beam at the absorber was $100 \mathrm{~W}$ [7]. Second, the absorbers must be located on the right and left sides in the horizontal plane and also have sufficient longitudinal length. Because the range of a $400 \mathrm{MeV}$ proton in oxygen-free copper is approximately $130 \mathrm{~mm}$, the necessary absorber length was determined to be $200 \mathrm{~mm}$, including a reasonable margin. Third, the beam side of the absorbers must have an angle. The schematic drawing is shown in Fig. 6. If the absorber is a rectangular parallelepiped, the horizontally spread scattered particles collide with the side of absorber. In this case, a sufficient flight distance in the absorber is not maintained, and the particles cannot be absorbed well. Therefore, the absorbers were designed with an angle such that the scattered particles collide in front of the absorber. Finally, the position and angle of the absorbers must be adjustable, because the beam width and scattered particle angle at the absorber are changed if the operational parameters are changed. Therefore, the absorber's support rods were designed to be adjustable through expansion and contraction. In addition, the absorbers were also designed to be rotatable around pivots located at the downstream end of the absorbers.

\section{ANGULAR REGULATION OF THE ABSORBERS}

\section{A. Construction of the simulation model}

To examine the localization effect, particularly of the absorbers, and the importance of the absorber angle, a simulation model was constructed using the beam tracking code SAD [16] for the beam tracking and GEANT4 for the scattering at the charge-exchange foil and particle reactions with the absorbers. In particular, the absorber structure and adjustment mechanism were constructed in detail in GEANT4. Using this model, a simulation of the distribution of losses between the injection point and the ring collimator was performed.

The large-angle scattering that needs to be eliminated has a very low probability of occurrence (approximately $10^{-5}$ ). Given this fact, some simplifications were applied to obtain sufficient statistics. Specifically, two simulation models were used for large-angle scattering, as described below.

The first model was a "uniform scattering simulation." From the results of the foil-scattering simulation, it became clear that the angular distribution of particles that have to be removed at the absorbers is nearly uniform. Therefore, the tracking of particles was performed with additional uniform horizontal angles at the injection point. This simulation was mainly used to estimate the particles that are created in and leak from the absorbers.

The second simulation was a "simple-multiturn simulation." This model considered the distribution of the scattering angle at the foil and that of the beam on the foil, which change during multiturn injection. The method is as follows. First, a separate multiturn injection simulation was performed by neglecting foil scattering, and the distribution of the beam on the foil was determined for each turn. Next, scattering angles greater than $\pm 3 \mathrm{mrad}$, which were obtained from the GEANT4 simulation, were added to this distribution, and the tracking was performed. In other words, the beams that did not relate to the losses between the foil and ring collimator were omitted by neglecting the decrease in the particles caused by the large-angle scattering. This simulation was mainly used to investigate the beam-based angular regulation.

In addition, the simulation area was restricted to the ring collimator, which collects the particles that are distant from the stable region. With these simplifications, the calculation time was drastically reduced.

\section{B. Dependence of the localization effect on the absorber angle}

The dependence of the localization effect on the absorber angle was examined by a uniform scattering simulation. A simulation of the protons that collide with the right-hand side absorber and the resulting leakage was performed. The kinetic energy of the protons was $400 \mathrm{MeV}$. The results are shown in Fig. 7.

The right-hand side absorber was inserted to reduce the losses located at the inside of the ring. These results indicated that, when the scattered particles and the beam side of the absorber were parallel, the losses were efficiently reduced. On the other hand, when the scattered particles and the beam side of the absorber were not parallel, the losses remained, because the particles that collided with the absorber leaked. These results confirmed the concept of the absorber design and indicated that the angular regulation of the absorbers is important. 


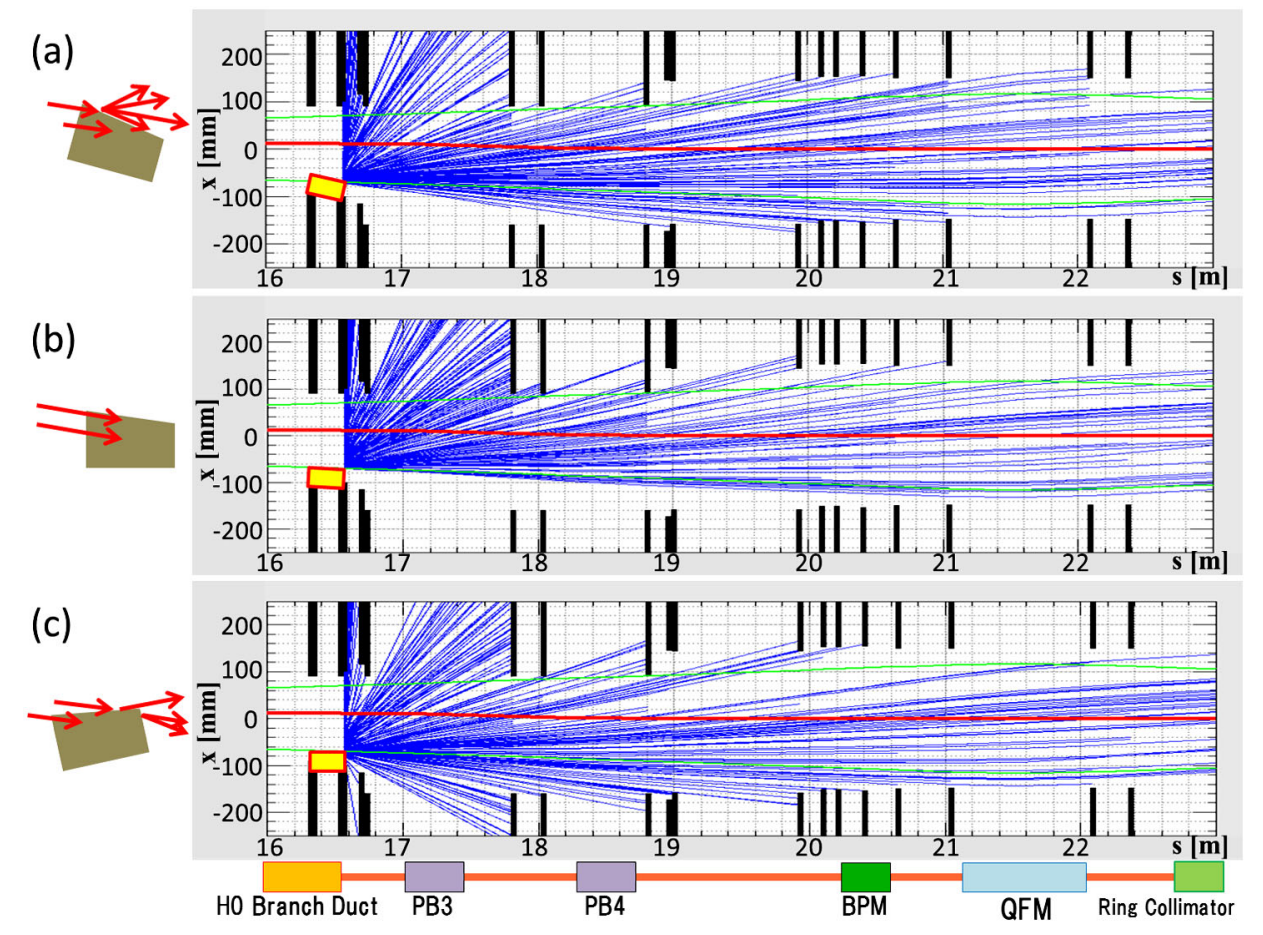

FIG. 7. Tracking of the particles leaking from the absorber between the absorber and the ring collimator: (a) rotated $1^{\circ}$ toward the beam center; (b) no rotation; (c) rotated $1^{\circ}$ away from the beam center.

In addition to dependence of the localization effect on the angle, it became clear that some of the particles colliding with the absorber leaked to the opposite side. However, the result of Fig. 7 indicates that the amount of this leakage was approximately $10 \%$ of the colliding particles. In other words, when the inside and outside absorbers are inserted enough to remove the losses between the absorbers and ring collimator and optimized as shown in Fig. 7(b), a maximum of $90 \%$ of the particles lost between the absorbers and the ring collimator will be able to localize at the absorbers.

\section{Method for the angular regulation of the absorber}

As described above, it was determined that the largeangle scattered particles and the sides of the absorbers should be aligned in parallel to efficiently localize the losses occurring between the absorber and QFM. Therefore, it was necessary to develop a method for the angular regulation of the absorber. In particular, it was desirable that the method be based on the actual beams, instead of calculations using the accelerator model. Thus, we explored the method using a simple-multiturn simulation. As a result, a method was devised for regulating the angle of the absorbers called the loss monitor method (LMM). The details of this method are explained using Fig. 8 as follows.

From this simulation, it became clear that when losses occurring at the inside of the BPM were intentionally left by not completely inserting the right-hand side absorber, and the absorber was rotated, a characteristic loss dependence with respect to rotation at the inside of the BPM could be determined. This loss dependence is shown in the right-hand part of Fig. 8 and can be explained as follows. Because the axis of rotation of the absorber is downstream, the inner edge downstream of the absorber is nearly fixed. In case 2 of Fig. 8, the scattered particles and the beam side of the absorber are parallel. In case 1, the loss decreases in comparison with that of case 2 , because the upstream edge of the absorber approaches the beam

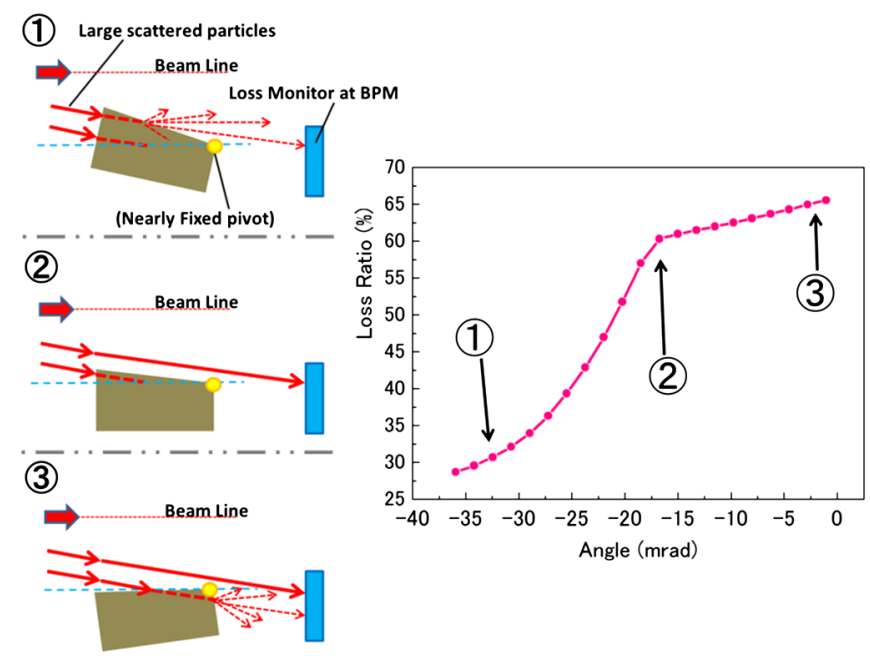

FIG. 8. Details of the LMM. The left figures show schematics of the rotation of the absorber placed on the inside of the ring. The right figure shows the loss dependence on the absorber rotation at the inside of the BPM. 
center, and more particles collide. On the other hand, in case 3 , the loss increases gradually in comparison with that in case 2, because the particles that collide with the front of the absorber in case 2 collide with the side of the absorber and leak out in case 3. Overall, these results indicate that the inflection point of the loss dependence on the absorber rotation is the absorber angle that is the same as that of the scattered particles. The LMM, therefore, is an effective method for determining the inflection point using a beam loss monitor at the BPM.

In addition, the loss dependence on the angle of the absorber between the absorber and the QFM was examined, and the results confirmed that not only a sufficient flight length in the absorber can be maintained for the scattered particles, but also the losses occurring between the absorber and the QFM can be minimized by optimization using the LMM.

It should be noted that, as a matter of course, an additional insertion after the optimization of the angle using the LMM is required to completely eliminate the losses.

\section{RESULTS OF THE BEAM TEST}

\section{A. Experimental method}

The loss reduction between the $\mathrm{H}^{0}$ collimation system and the QFM was then examined after insertion of the absorber. Ten plastic scintillators were placed horizontally as a beam loss monitor (BLM) between the $\mathrm{H}^{0}$ collimator and the QFM, and a BLM signal was obtained for $2 \mathrm{~ms}$ after the initiation of multiturn injection. The arrangement of the BLM is shown in Fig. 9. The value of the integration of the BLM signal from the initiation of injection to $700 \mu \mathrm{s}$ is defined as the loss caused by foil scattering, because the fixed orbit bump made by the SB1-4 begins to decay after the completion of multiturn injection, and the circulating beams hit the foil during approximately the first $700 \mu \mathrm{s}$, although the multiturn injection time is $500 \mu \mathrm{s}$. In addition, the value of the integration of the BLM signal from $700 \mu \mathrm{s}$ to $2 \mathrm{~ms}$ is defined as the loss caused by the halo of the circulating beams. Because this loss is originally localized at the ring collimator, this does not fundamentally exist between the $\mathrm{H}^{0}$ collimator and the QFM. However,

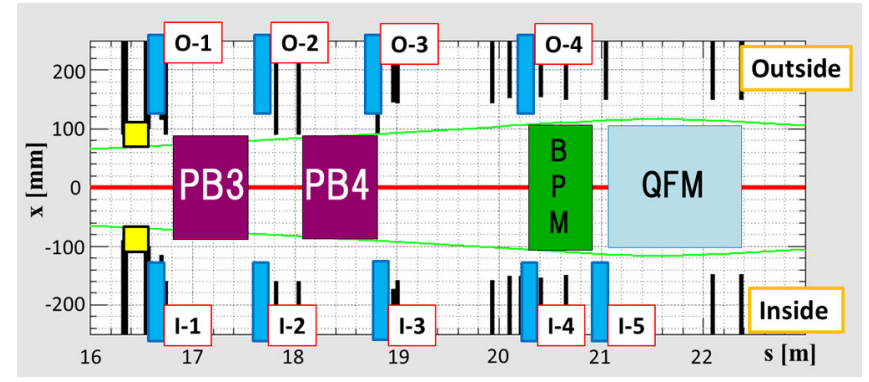

FIG. 9. Arrangement of the BLM.
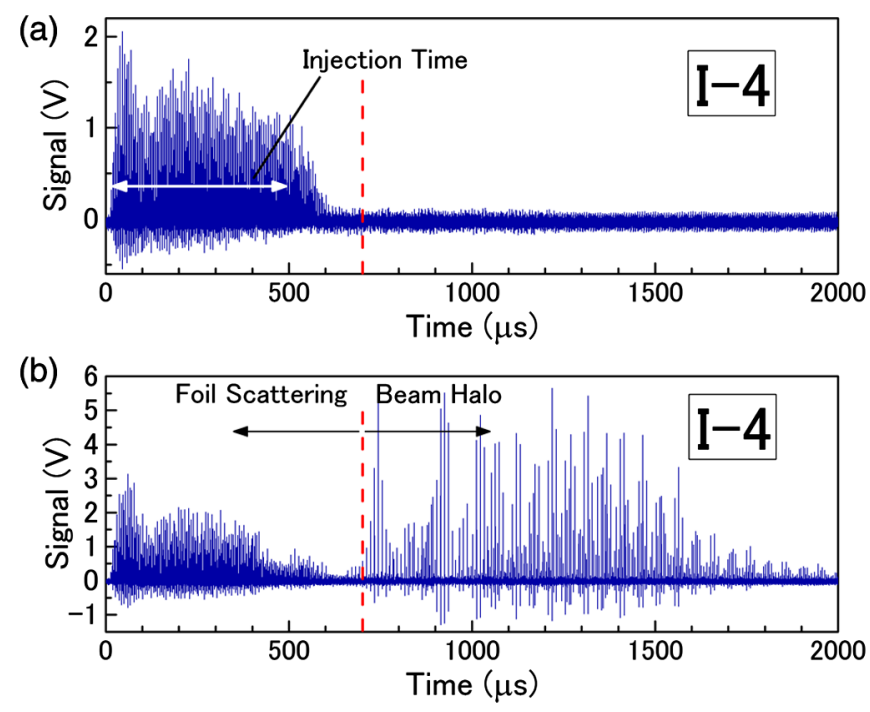

FIG. 10. A typical BLM signal during the injection period. The horizontal axis represents time, and the vertical axis represents the signal amplitude: (a) inside and outside absorbers retracted; (b) outside absorber inserted too far.

when the absorber is inserted too far, it behaves like the ring collimator and losses occur. A typical BLM signal is shown in Fig. 10.

\section{B. Effect of absorber insertion}

Next, the reduction of the loss between the $\mathrm{H}^{0}$ collimator and the QFM due to the insertion of the absorber was examined. During this investigation, the beam power was suppressed to approximately $1 / 10$ of the user operation level, and each absorber was inserted in turn. The results for the insertion of the absorber on the inside of the ring are shown in Fig. 11. As can be seen in the figure, it was confirmed that the losses caused by foil scattering on the inside of the BPM were suppressed by inserting the absorber. In addition, the increase in the losses caused by leakage from the opposite side of the absorber (e.g., at O-2) was minor. On the other hand, the losses caused by the halo increased on the other side of the inserted absorber when the absorber was inserted too far. From these results, the limit for the insertion of the absorber was determined.

In the same manner, results for the insertion of the second absorber were obtained, and it was confirmed that the loss caused by foil scattering at the outer circumference of the PB4 was suppressed by inserting this absorber.

\section{Optimization of the absorber angle}

As a next step, the possibility of optimizing the absorber angle using the LMM obtained from the simulation results was investigated. The amount of insertion of the absorbers was adjusted to retain some losses at 

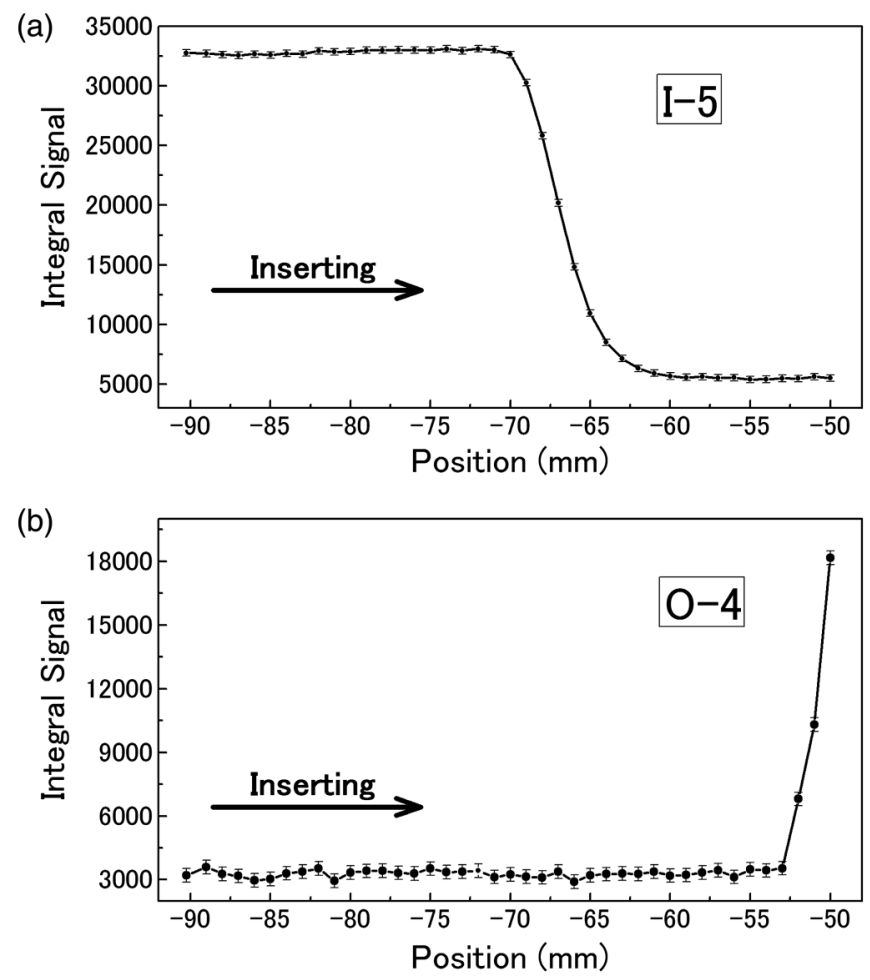

FIG. 11. Loss dependence on the insertion of the inside absorber at (a) I-5 and (b) O-4. The horizontal axis corresponds to the position of the absorber. The vertical axis corresponds to (a) the loss caused by foil scattering, and (b) the loss caused by a halo of circulating beams.

the inner circumference of the BPM or the outer circumference of the PB4. The variation of losses at these points while rotating the absorber was then detected. The results obtained for the rotation of the absorber located at the inside of the ring are shown in Fig. 12. As can be seen in the figure, the losses measured at the inside of the BPM had an inflection point. Moreover, it was confirmed that the losses at other points were minimized when the angle of the absorber corresponded with this inflection point. These results are in agreement with those of the simulation. The same results were also obtained for the absorber located on the outside of the ring. Thus, it was confirmed that the LMM can be used for the optimization of the absorbers.

\section{Results of the user operation test}

Finally, the effect of the $\mathrm{H}^{0}$ collimator using the same beam parameters as those for $220 \mathrm{~kW}$ user operation was evaluated. In this test, the absorber was inserted sufficiently after optimization using the LMM.

The losses near PB3 increased by a factor of 1.4 due to insertion of the absorber, which caused a leakage of the particles that entered the absorber. However, the residual dose near PB3 was several tens of $\mu \mathrm{Sv} / \mathrm{h}$,
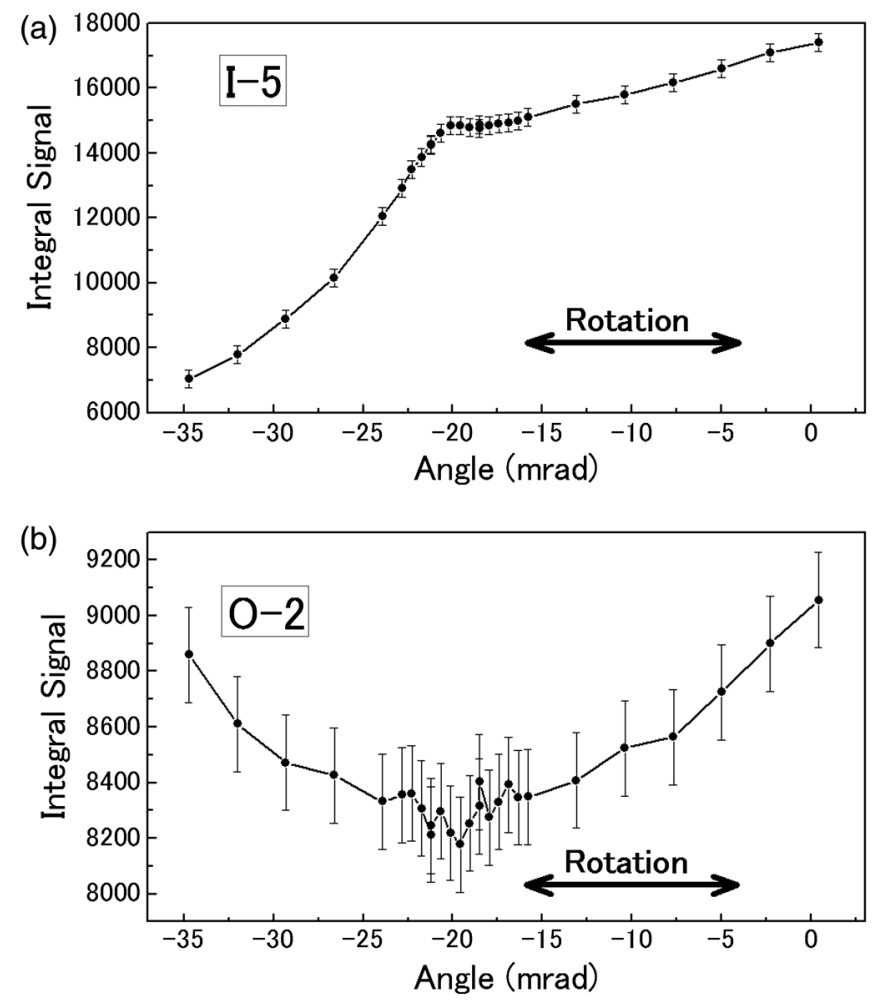

FIG. 12. Loss dependence on the angle of the inside absorber at (a) I-5 and (b) O-2. The horizontal axis corresponds to the angle of the absorber and the vertical axis corresponds to the loss caused by foil scattering.

which is small enough not to be a serious problem for maintenance.

The BLM signals at O-3 and I-5 are shown in Fig. 13. Based on these results, it can be seen that the losses occurring at the inside of the BPM and the outside of the PB4 decreased due to the insertion of the absorbers to $12 \%$ and $2 \%$, respectively, of the values when the absorbers were extracted. Therefore, the localization of the losses between the $\mathrm{H}^{0}$ branch and the QFM was achieved. In addition, the beam loss distribution of the entire RCS ring did not change after insertion of the absorbers.

The residual dose at the inside of the BPM was $230 \mu \mathrm{Sv} / \mathrm{h}$ after one operation cycle with the $\mathrm{H}^{0}$ collimator at a $120 \mathrm{~kW}$ output beam power. Because the residual dose was previously $4.5 \mathrm{mSv} / \mathrm{h}$ after one operation cycle at a $200 \mathrm{~kW}$, this value is approximately $1 / 10$ compared to that obtained without the collimator. On the other hand, the residual dose at the $\mathrm{H}^{0}$ branch could not be compared to the original value, because it was already covered by a radiation shield. However, the residual doses on the surface of the side of the radiation shield and upstream of the $\mathrm{H}^{0}$ branch that were not covered by a shield were sufficiently enough $(5 \mu \mathrm{Sv} / \mathrm{h}$ and $230 \mu \mathrm{Sv} / \mathrm{h}$, respectively) with the $\mathrm{H}^{0}$ collimator to enable maintenance of the RCS. 

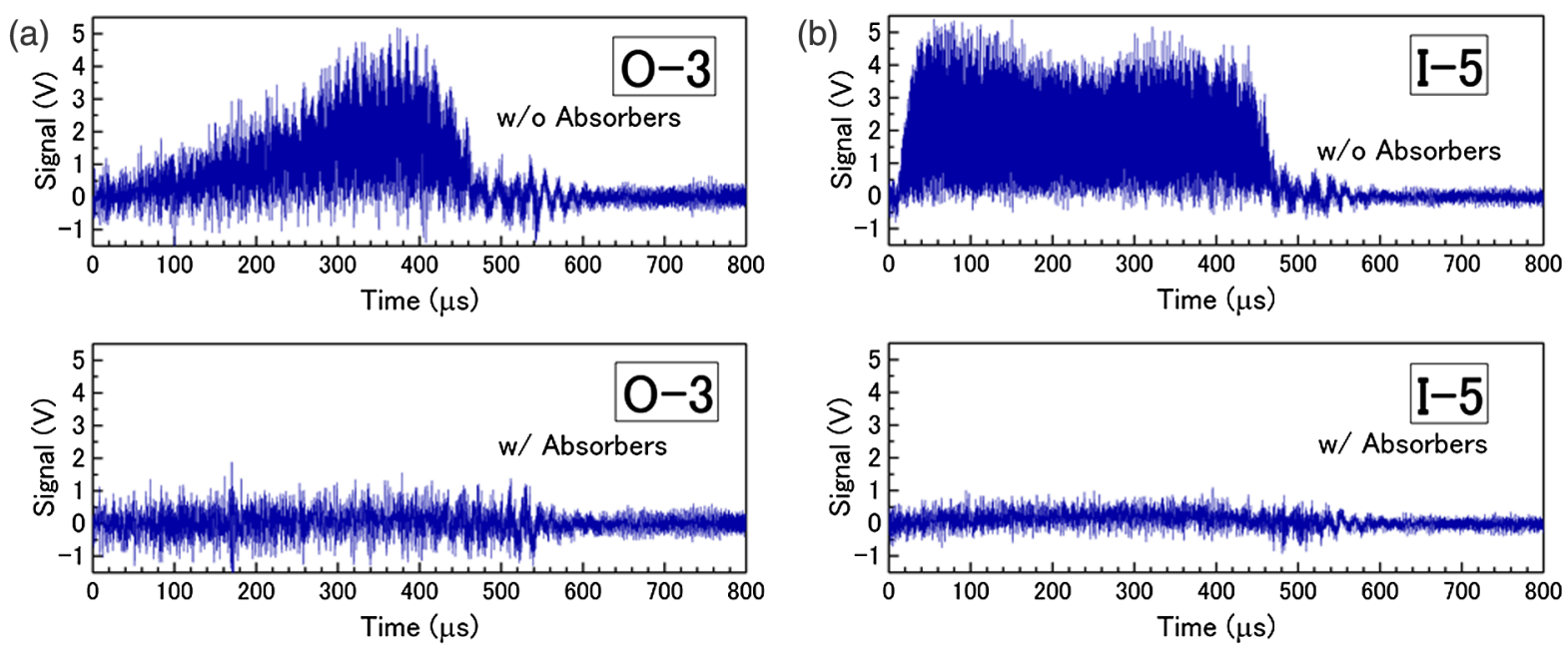

FIG. 13. BLM signal during the injection period at (a) O-3 and (b) I-5. The horizontal axis represents time, and the vertical axis represents the signal amplitude. Upper: extracting the absorbers. Lower: inserting the absorbers.

\section{CONCLUSION}

To realize a higher power operation at the $3 \mathrm{GeV} R C S$ of the J-PARC, a new collimation system (the $\mathrm{H}^{0}$ collimator) was developed to localize the losses between the $\mathrm{H}^{0}$ branch and the QFM caused by large-angle scattering of the injection and circulating beams at the charge-exchange foil during beam injection. The localization point and design of the absorbers were determined using the results of tracking simulations. In addition, the magnetic shielding effect and outgassing rate of the $\mathrm{H}^{0}$ branch duct were evaluated, and it was confirmed before construction that the duct had sufficient performance. A simulation model was then developed to examine the method for regulating the angle of the absorber. These results indicated that optimization of the angle of the absorbers is important, and that the LMM is useful for carrying out such optimization.

Finally, a beam test was conducted, and it was confirmed that the $\mathrm{H}^{0}$ collimator localized the losses, and that the angle of the absorber was optimized using the LMM. In addition, the residual doses were suppressed by the $\mathrm{H}^{0}$ collimator, and the values were sufficiently low to enable maintenance of the RCS. The beam losses between the $\mathrm{H}^{0}$ branch and the QFM and the residual doses were reduced to under one-tenth of those observed in the past. Therefore, the possibility of achieving a $1 \mathrm{MW}$ beam power in the RCS has become more realistic.

\section{ACKNOWLEDGMENTS}

The experimental data presented here were taken at the first machine study after the great East Japan earthquake. The authors would like to express their sincere gratitude to all of the J-PARC staff who worked inexhaustibly for rehabilitation.
[1] Y. Yamazaki et al., JAERI Report No. JAERI-Tech 2003044 and KEK Report No. 2002-13.

[2] H. Harada, KEK Report No. 2009-7.

[3] I. Sugai, Y. Takeda, M. Oyaizu, H. Kawakami, Y. Hattori, and K. Kawasaki, Nucl. Instrum. Methods Phys. Res., Sect. A 521, 192 (2004).

[4] H. Hotchi et al., Phys. Rev. ST Accel. Beams 12, 040402 (2009).

[5] P. K. Saha, in Proceedings of the 46th ICFA Advanced Beam Dynamics Workshop on High-Intensity and High-Brightness Hadron Beams, Morschach, Switzerland, 2010 (PSI, Morschach, 2010), pp 324-328.

[6] K. Yamamoto et al., in Proceedings of the 1st International Particle Accelerator Conference, Kyoto, Japan, 2010 (ICR, Kyoto, 2010), pp 2842-2844.

[7] K. Yamamoto et al., in Proceedings of the 2nd International Particle Accelerator Conference, San Sebastian, Spain, 2011 (EPS-AG, Spain, 2011), pp 1605-1607.

[8] http://geant4.cern.ch/.

[9] http://www-ap.fnal.gov/MARS/.

[10] M. Yoshimoto et al., in Proceedings of the 2nd International Particle Accelerator Conference, San Sebastian, Spain, 2011 (Ref. [7]), pp 1545-1547.

[11] S. Kato, J. Kamiya, K. Yamamoto, M. Yoshimoto, and M. Kinsho, J. Vac. Soc. Jpn. 55, 160 (2012).

[12] M. Yoshimoto, IEEE Trans. Appl. Supercond. 18, 297 (2008).

[13] N. Ogiwara, M. Kinsho, J. Kamiya, K. Yamamoto, M. Yoshimoto, Y. Hikichi, K. Kanazawa, K. Mio, Y. Takiyama, K. Suganuma, and Y. Saito, Vacuum 84, 723 (2009).

[14] http://www.san-ai-plant.co.jp/clean_technology/chemical_ polishing/sus_cp/.

[15] K. Yamamoto, Ph.D. thesis, The Graduate University for Advanced Studies, 2007.

[16] http://acc-physics.kek.jp/SAD/. 